\title{
Canadian Anesthesia Incident Reporting System (CAIRS): The Canadian Anesthesiologists' Society's National Patient Safety Initiative
}

\author{
W. Scott Beattie, MD, PhD, FRCPC • Martin D. Culwick, MBChB, FANZCA, MIT • \\ Hilary P. Grocott, MD, FRCPC, FASE
}

Received: 17 January 2018/Revised: 24 March 2018/Accepted: 16 April 2018/Published online: 27 April 2018

(C) Canadian Anesthesiologists' Society 2018

Anesthesia practice has made great strides to improve the delivery of safe anesthetic care. In a widely cited metaanalysis, Daniel Bainbridge and his colleagues documented a more than tenfold reduction in anesthesia-related mortality since the 1970s. ${ }^{1}$ Nevertheless, the failure of perioperative and other anesthesia-related patient safety initiatives remains a cause for concern in healthcare systems globally. The most recent data from the International Surgical Outcomes Trial (ISOS) show that more than $10 \%$ of patients suffer a moderate-to-severe adverse postoperative event. ${ }^{2,3}$ This adversity, be it surgical or anesthesia related, results in increased hospital stay and $\cos ^{4-6}$ as well as increasing negligence claims. ${ }^{7}$ The extent to which these adverse events are secondary to errors and/or a breakdown in processes of care, and therefore preventable, is unknown. ${ }^{8}$ There is a strong belief, however, that perioperative outcomes can be improved.

Presently, the majority of patient safety initiatives are anchored in the belief that safety can be improved by learning from critical incidents. ${ }^{9}$ This movement to indepth critical incident investigation stems directly from past demonstrations of improved outcomes after study of

W. Scott Beattie, MD, PhD, FRCPC ( $\square)$

University Health Network - Toronto General Hospital, 200

Elizabeth Street 3EN-464, Toronto, ON M5G 2C4, Canada

e-mail: scott.beattie@uhn.ca

M. D. Culwick, MBChB, FANZCA, MIT

Department of Anesthesia, Royal Brisbane and Women's

Hospital, Brisbane, QLD, Australia

H. P. Grocott, MD, FRCPC, FASE

Department of Anesthesia, Perioperative and Pain Medicine,

University of Manitoba, Winnipeg, MB, Canada similar near misses. For example, this type of introspective activity resulted in the improved safety and performance among military pilots in the Second World War. ${ }^{10}$ Indeed, critical incident analysis forms the basis of today's modern aviation safety management systems, ${ }^{11}$ which also now includes a proactive assessment of system hazards and flaws. The modern safety enhancement systems grew out of these earlier seminal initiatives. In a variety of companies, as diverse as $3 M$ to Amazon, documentation and a datadriven analysis of incidents allow for the adoption of diverse behaviours that serve to ameliorate undesirable outcomes.

In 1978, the notable patient safety advocate and Anesthesia Patient Safety Foundation co-founder, Jeffery Cooper, ${ }^{12}$ and his colleagues interviewed practicing anesthesiologists and obtained descriptions of preventable intraoperative incidents. Their article, entitled "Preventable Anesthesia Mishaps", was the first example of critical incident reporting in anesthesia practice $^{13}$ in which they estimated that over $80 \%$ of errors were preventable. Forty years later, in Canada, this activity is promoted in the Canadian Anesthesiologists' Society (CAS) Guidelines to the Practice of Anesthesia ${ }^{14}$ where, under the Department Chief duties, it states one of the primary responsibilities to be to:

"...employ a systematic approach for monitoring the quality of anesthetic care provided by members of the department of anesthesia throughout the healthcare facility; monitoring quality of care may include, but need not be restricted to, the use of chart audits, clinical indicator and outcome monitoring, adverse event reporting systems, morbidity and mortality conferences, and critical incident case reviews".

While the CAS promulgates this guideline, the frequency with which it occurs, veracity of the 
information acquired, and changes to practice and outcomes resulting from them are rarely shared at the national level. Unfortunately, the magnitude of improvements attributed to critical incident analysis seen in other industries has yet to be shown in perioperative medicine.

The World Health Organization (WHO) published the "Draft Guidelines for Adverse Event Reporting and Learning Systems" with a checklist for incident reporting systems. ${ }^{15}$ Simplified, the guideline calls for the inclusion of four steps:

i) Data input-which should be voluntary, confidential, non-punitive, and independent;

ii) Data collection - wherein it should be clear who can report, how a report should be filed, and what is expected to be reported;

iii) Data analysis - which should include a classification of events and a systematic approach to the analysis, be conducted by experts, and be reported in a timely and regular fashion;

iv) Feedback-which should contain recommendations and follow-up.

Finally, it should be obvious that all changes must also be monitored to ensure that the desired outcomes are achieved.

The wider implementation and collective global experience with clinical incident reporting systems have exposed several limitations. A recent meta-analysis has shown that only a few Incident Reporting Systems (IRS) employed in critical care settings contain all elements suggested by the WHO checklist. ${ }^{16}$ This analysis showed that the data input and collection systems are well established and aligned with the checklist. Nevertheless, among the major identified deficiencies of the existing IRS is the lack of a consistent approach to the analysis of incidents. Furthermore, there do not appear to be consistent mechanisms to provide feedback of information or the ability to effectively promote corrective actions. These deficiencies hinder any chance of achieving or rivalling the success of IRS seen in industry.

Even when all the components of the WHO guidelines are met, under-reporting of critical incidents hinders quality improvement. ${ }^{17,18}$ The Canadian Anesthesia Incident Reporting System (CAIRS) is based upon webAIRS, which is the IRS sponsored by the Australia New Zealand Tripartite Data Committee (ANZTADC). Despite the over 5,700 critical incidents that have been reported to webAIRS since $200^{9,19}$ this almost certainly represents a very small percentage of the anaesthetic incidents that have occurred in Australia and New Zealand over the past nine years. The reason for the relatively low
Table Abridged taxonomy for reportable incidents

\begin{tabular}{|c|c|}
\hline Main category & Examples of some subcategories \\
\hline Anesthetic related & $\begin{array}{l}\text { Airway management } \\
\text { Anaphylaxis } \\
\text { Aspiration } \\
\text { Awareness } \\
\text { Malignant hyperthermia } \\
\text { Regional anesthesia complications } \\
\text { Renal complications }\end{array}$ \\
\hline Respiratory & $\begin{array}{l}\text { Bronchospasm } \\
\text { Desaturation/hypoxia } \\
\text { Pneumothorax } \\
\text { Prolonged ventilation } \\
\text { Pulmonary edema } \\
\text { Unplanned re-intubation } \\
\text { Unplanned critical care admission } \\
\text { Ventilatory failure or arrest }\end{array}$ \\
\hline Cardiovascular & $\begin{array}{l}\text { Arrest } \\
\text { Need for support } \\
\text { Instability (dysrhythmias, hypo- or } \\
\quad \text { hypertension) } \\
\text { Myocardial ischemia or infarction } \\
\text { Vascular complications }\end{array}$ \\
\hline Medication & $\begin{array}{l}\text { Administration error } \\
\text { Allergy } \\
\text { Unavailable/shortage }\end{array}$ \\
\hline Equipment/device & $\begin{array}{l}\text { Malfunction } \\
\text { Unavailable }\end{array}$ \\
\hline Documentation & $\begin{array}{l}\text { Preoperative assessment } \\
\text { Postanesthesia care } \\
\text { Events }\end{array}$ \\
\hline $\begin{array}{l}\text { Process of care } \\
\text { failure }\end{array}$ & $\begin{array}{l}\text { Administrative related } \\
\text { Diabetes management } \\
\text { Fatigue related } \\
\text { Infection prophylaxis } \\
\text { Lack of skilled assistance } \\
\text { Lack of supervision } \\
\text { Handover/checklist } \\
\text { Hematology/transfusion/coagulation } \\
\text { NPO guidelines/preoperative medications } \\
\text { VTE prophylaxis }\end{array}$ \\
\hline Neurologic & $\begin{array}{l}\text { Blindness } \\
\text { CVA/stroke/coma } \\
\text { Peripheral nerve injuries } \\
\text { Paralysis } \\
\text { Seizure }\end{array}$ \\
\hline
\end{tabular}

$\overline{C V A \text { cerebrovascular accident, } N P O \text { nothing by mouth, VTE venous }}$ thromboembolism 
reporting rate might be the multiple disincentives to incident reporting. ${ }^{20,21}$ The major barriers include:

i) Reporting may be time consuming;

ii) Lack of easy access to the reporting system;

iii) The fear of reprisal and/or liability;

iv) Variability in the definition of a critical incident (i.e., lack of understanding about what should be reported);

v) A lack of feedback (i.e., many thought that the data were being entered into a "black box" with no analysis).

The Pediatric Anesthesia Group at Stanford have undertaken multiple interventions to improve reporting rates. ${ }^{19}$ Interventions included embedding the IRS within the electronic medical record (EMR), a series of interviews with faculty, and detailed feedback to the users. These steps lead to a significantly increased number of incidents being reported. The CAS and CAIRS plan to engage in these processes. Another major impediment to reporting is duplication. Many Canadian institutions have generic electronic incident reporting systems in place. The CAIRS technical team is currently investigating electronic solutions that seek to minimize the need for any user to report in duplicate.

The CAIRS has just been recently launched (15 March 2018) and has been designed be easy and accessible. The CAIRS is initially available to all members of the CAS at www.CAIRS.ca though the future plan is for it to be available to individual Departments of Anesthesia independent of CAS membership. This will create an even broader patient safety network allowing for uniform and in-depth perioperative safety reporting at both the local and national levels. This launch, and the ultimate design of CAIRS, is based on input from the experiences gathered from similar systems that are now in place in the United States, United Kingdom, Australia, and New Zealand. Importantly, CAIRS can be easily accessed from anywhere, on any internet-enabled platform. The intuitive navigation that CAIRS employs allows for easy data entry. The questions are open ended and aligned with other national IRS. The design can capture any event throughout the perioperative period. Data entry is confidential and can be anonymous; indeed, the choice of anonymity is left to the reporter, thus minimizing fear of reprisal.

Admittedly, defining a reportable incident or instructing users what to report is somewhat problematic. The object of CAIRS is to collect all incidents, errors, and complications whether or not the event leads to harm. The system, by design, has to be open-ended and nondirectional. Historically, critical incident reports have been overwhelmingly from the intraoperative period, but we must also remain vigilant for, and thus report, pre- and postoperative events. Similar reports show that $>75 \%$ of the reports are confined to airway-, respiratory-, medication-, cardiovascular-, and equipment-related incidents. ${ }^{15,22}$ We remind prospective users that it is also important to report process and documentation failure as well. Also, the great strength of any IRS is the reporting of infrequent events, such as neurologic injury and deaths. Though CAIRS does not instruct or point users to any type of critical incident, errors, or complications, an abridged taxonomy for reportable events is presented in the Table. We remind users that all incidents, however minor, are worthy of a report.

To address the most common shortcoming identified by the referenced critical care meta-analysis of IRS, the CAS and CAIRS are acting to ensure timely data reporting. As such, CAIRS employs a medical director charged with checking new entries on a weekly basis. An expert committee will perform adverse event analysis on a regular basis. The CAS is in the midst of a national search for a chair of the CAIRS analytic oversight committee. The mandate of the chairperson will be to oversee robust data-driven analysis and feedback. This type of root-cause analysis improves perioperative patient safety at the institutional level. ${ }^{23}$ An oversight committee will be staffed with clinical experts who can support feedback of information and improvement measures while assisting in the implementation of these measures. The make-up of the committee has not been set at this time, and we urge any and all interested members to make their desires known. The plan is also to approach the Association of Canadian University Departments of Anesthesia (ACUDA) for academic input in addition to an anesthesia trainee representative. Cognizant of the recent Canadian Medical Protective Association (CMPA) communications, it is also our intent to appoint a patient advocate to the committee. ${ }^{24}$ The CAS has numerous vehicles to distribute CAIRS feedback including the Journal, the CAS website, CAS newsletter, as well as through social media and direct email.

The launch of CAIRS comes with some potential concerns. It also comes after a close consultation process with ANZTADC as well as a rigorous liability consultation process. The CAS has obtained opinions from the CMPA and other select members of the legal community. Patients' privacy concerns have been addressed through a vetting process conducted by the University Health Network's privacy office. The CAIRS website is secure, anonymous, and available in both official languages. Finally, we have sought input from the CAS membership itself. A pilot version of CAIRS has been available to members since June 2017. The results of a questionnaire linked to this demonstration site suggest that members endorse the implementation of this National Incident Reporting System. More than $80 \%$ of responders stated they would 
use CAIRS when it comes online. Eighty-three percent felt that CAIRS would improve anesthesia practice in Canada. Although concern was expressed by approximately $50 \%$ of respondents that there was a potential for a breach in patient confidentiality and/or privacy, we can assure CAS members that all data are collected free of any patientidentifying features. Respondents rightly felt that embedding the IRS within an EMR would increase costs. The respondents were also concerned that funding (or lack thereof) for CAIRS had the potential to limit its penetration into widespread practice. The Australian and New Zealand College of Anaesthetists, Australian Society of Anaesthetists (Australian ASA), and New Zealand Society of Anaesthetists jointly fund ANZTADC. Over $66 \%$ of respondents to our survey stated they would be willing to support CAIRS through the CAS in a fashion similar to how webAIRS is supported by its component societies.

In conclusion, CAIRS was developed to comply with the WHO recommendations related to perioperative safety. The software was designed to be a robust, easily accessible, and intuitive critical perioperative incident-capturing tool. Furthermore, CAIRS is confidential (and can be anonymous), ensures patient privacy, and can be accessed from any internet-enabled platform. The data and the ensuing recommendations will be evaluable by a national panel of experts. We are excited by the prospects for the future and ask for the continued feedback and support of CAS members and other interested readers.

\section{Système de déclaration \\ canadien des incidents \\ d'anesthésie (CAIRS) : \\ l'initiative nationale de \\ sécurité des patients de la \\ Société canadienne des anesthésiologistes}

La pratique de l'anesthésie s'est efforcée d'améliorer la sécurité de ses soins. Dans une méta-analyse souvent citée, Daniel Bainbridge et ses collègues ont documenté une baisse de la mortalité par un facteur supérieur à 10 depuis les années 1970. ${ }^{1}$ Toutefois, l'échec des initiatives concernant la sécurité des patients en période périopératoire ou en rapport avec l'anesthésie reste, dans le monde, une préoccupation majeure des soins de santé. Les données les plus récentes provenant de l'essai ISOS (International Surgical Outcomes Trial) montrent que plus de $10 \%$ des patients présentent un événement indésirable postopératoire modéré à sévère. ${ }^{2,3}$ Cet échec, qu'il soit en rapport avec la chirurgie ou l'anesthésie, entraîne un allongement des hospitalisations et de leur coût, ${ }^{4-6}$ ainsi qu'une augmentation du nombre de plaintes pour négligence. ${ }^{7}$ On ignore dans quelle mesure ces événements indésirables sont secondaires à des erreurs et/ ou à des ratés des processus de soins (les rendant donc évitables). ${ }^{8}$ Néanmoins, pour beaucoup, ces résultats périopératoires peuvent être améliorés.

Actuellement, la majorité des initiatives portant sur la sécurité des patients est ancrée dans l'idée que la sécurité peut être améliorée si l'on tire la leçon des incidents majeurs. 9 Ce mouvement en faveur d'une enquête approfondie sur les incidents majeurs repose sur des démonstrations anciennes de l'amélioration des résultats après l'étude d'erreurs quasi identiques. Ce type d'introspection a permis, par exemple d'améliorer l'efficacité et les performances des pilotes militaires au cours de la Deuxième Guerre mondiale. ${ }^{10}$ L'analyse des incidents majeurs constitue la base des systèmes modernes de gestion de la sécurité dans l'aviation, ${ }^{11}$ ce qui inclut aussi maintenant une évaluation proactive des dangers et insuffisances des systèmes. Les systèmes modernes d'amélioration de la sécurité se sont développés à partir de ces concepts. Dans un grand nombre d'entreprises aussi différentes que $3 M$ et Amazon, la documentation et l'analyse des incidents basés sur les données permettent d'adopter différents comportements qui servent à améliorer les résultats indésirables.

En 1978, le défenseur bien connu de la sécurité des patients et cofondateur de la fondation pour la sécurité anesthésique des patients (APSF - Anesthesia Patient Safety Foundation), Jeffery Cooper, ${ }^{12}$ et ses collègues ont interrogé des anesthésiologistes actifs en pratique clinique pour obtenir la description d'incidents peropératoires évitables. Leur article, intitulé «Preventable Anesthesia Mishaps », dans lequel il estimait que plus de $80 \%$ des erreurs étaient évitables, a été le premier exemple de rapport d'incident critique en pratique anesthésique. ${ }^{13}$ Quarante ans plus tard, au Canada, cette activité est promue dans les Lignes directrices pour la pratique de l'anesthésie de la Société canadienne des anesthésiologistes (SCA $)^{14}$ qui indique que l'une des principales responsabilités d'un chef de département doit être « [d']avoir recours à une approche systématique pour surveiller la qualité des soins anesthésiques offerts par les membres du département d'anesthésie à la grandeur de l'établissement de soins de santé. La surveillance de la qualité des soins peut comprendre, sans cependant s'y limiter, le recours à des vérifications de dossiers, la surveillance d'indicateurs et de suivis cliniques, les systèmes de surveillance des événements indésirables, 
des conférences sur la morbidité et la mortalité, et la révision des cas d'incidents critiques. »

Bien que la SCA ait publié ces lignes directrices, la fréquence de survenue des incidents critiques, la véracité de l'information acquise ainsi que les modifications dans les pratiques et résultats qui en découlent sont rarement communiqués au niveau national. Malheureusement, l'ampleur des améliorations attribuées à l'analyse des incidents critiques, telle qu'on la voit dans d'autres secteurs d'activité, reste encore à démontrer en médecine périopératoire.

L'Organisation mondiale de la santé (OMS) a publié les «Directives sur les systèmes de notification des effets indésirables et d'apprentissage » avec une liste de contrôle pour les systèmes de déclaration des incidents. ${ }^{15}$ Simplifiées, les lignes directrices demandent l'inclusion de quatre étapes :

i) La saisie des données - qui doit être volontaire, confidentielle, non punitive et indépendante;

ii) La collecte de données - pour laquelle il doit être clairement établi qui peut faire le rapport, comment ce dernier doit être rempli et ce qu'on s'attend à voir déclaré;

iii) L'analyse des données - qui doit inclure un classement des événements et une démarche d'analyse systématique, menée par des experts et publiée dans des délais opportuns et de façon régulière;

iv) Une rétroaction - qui doit contenir des recommandations et un suivi.

Enfin, il devrait être évident que toutes les modifications doivent aussi être contrôlées pour s'assurer que les résultats désirés ont été obtenus.

Une mise en œuvre plus large et une expérience collective internationale des systèmes de déclaration des incidents cliniques ont fait apparaître plusieurs limites. Une méta-analyse récente a montré que seulement quelques systèmes de déclaration des incidents (IRS - Incident Reporting Systems) utilisés dans les cadres de soins intensifs incluaient tous les éléments suggérés par la liste de vérification de l'OMS. ${ }^{16}$ Cette analyse a montré que la saisie des données et les systèmes de collecte sont bien établis et correspondent à la liste de vérification. Néanmoins, parmi les faiblesses majeures identifiées dans les IRS existants, on note l'absence de démarche cohérente d'analyse des incidents et il ne semble pas exister de mécanismes cohérents visant à fournir un retour d'information ou une capacité à promouvoir efficacement des actions correctrices. Ces défauts limitent toute chance d'atteindre ou de rivaliser avec le succès des IRS en place dans l'industrie.
Même lorsque tous les éléments des lignes directrices de l'OMS sont présents, la sous-déclaration des incidents critiques gêne l'amélioration de la qualité. ${ }^{17,18}$ Le Système canadien de déclaration des incidents d'anesthésie (CAIRS) repose sur webAIRS qui est l'IRS promu par l'ANZTADC (Comité tripartite Australie/Nouvelle-Zélande sur les données). Plus de 5700 incidents critiques ont été déclarés sur webAIRS depuis $200^{9,19}$ mais ils ne représentent presque certainement qu'un très faible pourcentage de tous les incidents d'anesthésie survenus en Australie en Nouvelle-Zélande au cours des neuf dernières années. La raison de ce relativement faible taux de déclaration pourrait être les multiples éléments décourageant la déclaration des incidents. ${ }^{20,21}$ Ces principaux obstacles sont, notamment :

vi) Le fait que la déclaration peut prendre du temps;

vii) L'absence d'accès simple au système de déclaration;

viii) La peur de représailles et/ou de mise en cause;

ix) La variabilité de la définition d'un incident critique (c'est-à-dire le manque de compréhension sur ce qui doit être déclaré);

x) Une absence de rétroaction (beaucoup ont estimé que les données étaient saisies dans un « trou noir » sans analyse).

Le groupe d'anesthésies pédiatriques de Stanford a entrepris de multiples interventions pour améliorer les taux de déclaration. ${ }^{19}$ Ses interventions ont inclus, notamment, l'inclusion de l'IRS dans le dossier médical électronique (DME), une série d'entretiens avec le corps professoral et une rétroaction détaillée aux utilisateurs. Ces étapes ont conduit à une augmentation significative du nombre d'incidents déclarés. La SCA et le CAIRS prévoient de se lancer dans ces processus. Un autre problème majeur des déclarations est constitué par la duplicité. De nombreux établissements canadiens disposent de systèmes électroniques de création de rapports d'incident. L'équipe technique du CAIRS recherche actuellement des solutions électroniques visant à minimiser le besoin pour tout utilisateur de faire une déclaration en double.

Le CAIRS vient seulement d'être lancé (15 mars 2018) et a été conçu dans une optique de simplicité et d'accessibilité. Le CAIRS est initialement à la disposition de tous les membres de la SCA à l'adresse www.CAIRS.ca bien que le plan futur prévoie de le mettre à la disposition de chaque département d'anesthésie indépendamment de son affiliation à la SCA. Cela créera un réseau encore plus vaste pour la sécurité des patients, offrant un système de déclaration de la sécurité périopératoire, uniforme et approfondi, autant au niveau local qu'au niveau national. Ce lancement, et le projet ultime du CAIRS, se base sur les expériences acquises avec des systèmes similaires qui sont 
maintenant en place aux États-Unis, au Royaume-Uni, en Australie et en Nouvelle-Zélande. Il est important de rappeler que le CAIRS est accessible de partout et à partir de n'importe quelle plateforme ayant accès à l'Internet. Le mode de navigation intuitive que le CAIRS emploie permet une saisie facile des données. Les questions sont ouvertes et compatibles avec les autres IRS nationaux. Le concept peut saisir tout événement intervenant au cours de l'ensemble de la période périopératoire. La saisie des données est confidentielle et peut-être anonyme; le choix de l'anonymat est laissé à la personne faisant la déclaration, réduisant ainsi la crainte de représailles.

Il est vrai que définir un incident à déclarer ou indiquer aux utilisateurs ce qu'ils doivent déclarer peut poser un problème. L'objectif du CAIRS est de collecter tous les incidents, les erreurs et complications, que cet événement aboutisse à un préjudice ou non. Pour cela, la conception du système doit être ouverte et non directive. Historiquement, les rapports d'incidents critiques concernaient très largement la période peropératoire, mais nous devons rester vigilants pour ce qui concerne les événements pré et postopératoires, et donc les déclarer. Des rapports semblables montrent que plus de $75 \%$ des déclarations se limitent à des incidents en rapport avec les voies respiratoires, le système respiratoire, les médicaments, le système cardiovasculaire et l'équipement. ${ }^{15,22}$ Nous rappelons aux utilisateurs futurs qu'il est aussi important de déclarer les échecs d'un processus et les défauts de documentation. La grande force de tout IRS repose également sur la déclaration d'événements peu fréquents tels que les lésions neurologiques et les décès. Bien que le CAIRS ne donne pas de consignes ou n'oriente pas les utilisateurs vers un type particulier d'incident critique, d'erreur ou de complication, une taxonomie abrégée des événements déclarables est présentée dans le tableau. Nous rappelons aux utilisateurs que tous les incidents, même mineurs, valent la peine d'une déclaration.

Pour répondre à la difficulté la plus fréquente identifiée par la méta-analyse référencée sur les soins intensifs, la SCA et le CAIRS agissent pour assurer la déclaration de données en temps opportun. À ce titre, le CAIRS a un directeur médical chargé de contrôler chaque semaine les nouvelles entrées et un comité d'expert fera régulièrement l'analyse des événements indésirables. La SCA recherche actuellement au pays un président du comité de supervision analytique du CAIRS. Le mandat de ce responsable sera de superviser une analyse basée sur des données solides et la rétroaction. Ce type d'analyse de la cause (« root-case analysis ») améliore la sécurité périopératoire du patient au niveau de l'établissement. ${ }^{23}$ Un comité de supervision sera formé d'experts cliniques pouvant supporter le processus de rétroaction et les mesures d'amélioration tout en
Tableau Taxonomie abrégée des incidents déclarables

\begin{tabular}{|c|c|}
\hline Catégorie principale & Exemples de sous-catégories \\
\hline Liés à l'anesthésie & $\begin{array}{l}\text { Gestion des voies respiratoires } \\
\text { Anaphylaxie } \\
\text { Aspiration } \\
\text { Réveil sous anesthésie générale } \\
\text { Hyperthermie maligne } \\
\text { Complications de l'anesthésie régionale } \\
\text { Complications rénales }\end{array}$ \\
\hline Système respiratoire & $\begin{array}{l}\text { Bronchospasme } \\
\text { Désaturation/Hypoxie } \\
\text { Pneumothorax } \\
\text { Ventilation prolongée } \\
\text { Edème pulmonaire } \\
\text { Réintubation non prévue } \\
\text { Admission non prévue en unité de soins intensifs } \\
\text { Échec ou arrêt ventilatoire }\end{array}$ \\
\hline Cardiovasculaire & $\begin{array}{l}\text { Arrêt cardiaque } \\
\text { Besoin de soutien } \\
\text { Instabilité (troubles du rythme, hypo- ou } \\
\text { hypertension) } \\
\text { Ischémie ou infarctus myocardique } \\
\text { Complications vasculaires }\end{array}$ \\
\hline Médicaments & $\begin{array}{l}\text { Erreur d'administration } \\
\text { Allergie } \\
\text { Non-disponibilité/pénurie }\end{array}$ \\
\hline Matériel/dispositif & $\begin{array}{l}\text { Dysfonctionnement } \\
\text { Indisponible }\end{array}$ \\
\hline Documentation & $\begin{array}{l}\text { Évaluation préopératoire } \\
\text { Soins post anesthésie } \\
\text { Événements }\end{array}$ \\
\hline $\begin{array}{l}\text { Échec du processus } \\
\text { de soins }\end{array}$ & $\begin{array}{l}\text { Liés à l'administration } \\
\text { Gestion du diabète } \\
\text { Liés à la fatigue } \\
\text { Prophylaxie de l'infection } \\
\text { Manque d'aide compétente } \\
\text { Manque de supervision } \\
\text { Aide-mémoire/documents remis/liste de contrôle } \\
\text { Hématologie/transfusion/coagulation } \\
\text { Lignes directrices concernant le jeûne/les } \\
\text { médicaments préopératoires } \\
\text { Prophylaxie de la MTEV }\end{array}$ \\
\hline Système nerveux & $\begin{array}{l}\text { Cécité } \\
\text { AVC/coma } \\
\text { Lésions des nerfs périphériques } \\
\text { Paralysie } \\
\text { Crise comitiale }\end{array}$ \\
\hline
\end{tabular}

$A V C$ accident cérébrovasculaire, MTEV maladie thromboembolique veineuse

contribuant à la mise en œuvre de ces mesures. La constitution du comité n'a pas encore été définie à ce jour et nous invitons ardemment tous les membres intéressés à 
s'exprimer. Le projet consiste également à approcher l'association des départements universitaires canadiens d'anesthésie (ACUDA) pour avoir un avis universitaire en plus d'un représentant des résidents en anesthésie. Connaissant les communications récentes de l'ACPM (Association canadienne de protection médicale), nous avons également l'intention de nommer un représentant des patients à ce comité. ${ }^{24}$ La SCA dispose de nombreux supports pour transmettre le retour d'information du CAIRS, y compris le Journal, le site Web de la SCA, les médias sociaux et les courriels envoyés directement.

Le lancement du CAIRS s'accompagne de quelques préoccupations potentielles. Il a également lieu après un processus d'étroites consultations avec l'ANZTADC ainsi qu'un processus rigoureux de consultations sur la responsabilité. La SCA a recueilli différents avis de l'ACPM et d'autres membres choisis de la communauté de juristes. Les préoccupations concernant la confidentialité des patients ont été abordées via un processus de validation mené par le bureau d'accès à l'information confidentielle de l'UHN (University Health Network). Le site Web du CAIRS est sécurisé, anonyme et disponible dans les deux langues officielles. Enfin, nous avons demandé leur avis aux membres de la SCA. Les membres disposent d'une version pilote du CAIRS depuis juin 2017. Les résultats d'un questionnaire lié à ce site de démonstration suggèrent que les membres soutiennent la mise en œuvre du Système national de déclaration des incidents. Plus de $80 \%$ des personnes ayant répondu ont déclaré qu'ils utiliseraient le CAIRS quand il serait en ligne. Quatre-vingt-trois pour cent ont estimé que le CAIRS améliorerait la pratique de l'anesthésie au Canada. Bien que des préoccupations aient été exprimées par environ $50 \%$ des répondants à propos du risque de violation de la confidentialité et/ou de la vie privée des patients, nous pouvons assurer aux membres de la SCA que toutes les données sont collectées sans critères permettant d'identifier des patients. Les répondants ont estimé à juste titre que l'inclusion de l'IRS dans un DME augmenterait les coûts. Ils ont été également préoccupés par le fait que le financement (ou l'absence de financement) du CAIRS pourrait limiter sa pénétration dans une pratique plus généralisée. L'ANZTADC est financé conjointement par le Collège des anesthésiologistes d'Australie et de Nouvelle-Zélande, la Société australienne des anesthésiologistes (ASA australienne) et la Société néo-zélandaise des anesthésiologistes. Plus de $66 \%$ des personnes ayant répondu à notre enquête ont déclaré qu'ils seraient d'accord pour soutenir le CAIRS via la SCA de la même façon que webAIRS est soutenu par les sociétés qui le composent.

Pour conclure, le CAIRS a été développé pour se conformer aux recommandations de l'OMS concernant la sécurité périopératoire. Le logiciel a été conçu pour faire preuve de robustesse, offrir un accès facile et être un outil intuitif de saisie des incidents critiques périopératoires. Enfin, le CAIRS est confidentiel (et peut être anonyme), garantissant la confidentialité des patients; il est accessible depuis toute plateforme ayant accès à l'Internet. Les données et les recommandations qui en découleront seront évaluables par un comité national d'experts. Nous sommes enthousiasmés par un tel développement futur et nous demandons aux membres de la SCA et aux autres lecteurs intéressés de nous faire part en permanence de leurs points de vue et de leur soutien.

Conflicts of interest Drs. Beattie and Culwick have received remuneration for their roles in the development, testing, and deployment of CAIRS and webAIRS respectively.

Editorial responsibility This submission was handled by Dr. Gregory L. Bryson, Deputy Editor-in-Chief, Canadian Journal of Anesthesia.

Conflits d'intérêts Le Dr Beattie et le Dr Culwick ont reçu des rémunérations pour leurs rôles dans l'élaboration, les tests et le déploiement, respectivement, du CAIRS et du webAIRS.

Responsabilité éditoriale Cet article a été traité par le $\mathrm{D}^{\mathrm{r}}$ Gregory L. Bryson, rédacteur en chef adjoint, Journal canadien d'anesthésie.

\section{References}

1. Bainbridge D, Martin J, Arango M, Cheng D. Evidence-based Peri-operative Clinical Outcomes Research Group. Perioperative and anaesthetic-related mortality in developed and developing countries: a systematic review and meta-analysis. Lancet 2012; 380: 1075-81.

2. Ahmad T, Bouwman RA, Grigoras I, et al. Use of failure-torescue to identify international variation in postoperative care in low-, middle- and high-income countries: a 7-day cohort study of elective surgery. Br J Anaesth 2017; 119: 258-66.

3. Kahan BC, Koulenti D, Arvaniti K, et al. Critical care admission following elective surgery was not associated with survival benefit: prospective analysis of data from 27 countries. Intensive Care Med 2017; 43: 971-9.

4. Vonlanthen $R$, Slankamenac K, Breitenstein $S$, et al. The impact of complications on costs of major surgical procedures: a cost analysis of 1200 patients. Ann Surg 2011; 254: 907-13.

5. Davenport DL, Henderson WG, Khuri SF, Mentzer RM Jr. Preoperative risk factors and surgical complexity are more predictive of costs than postoperative complications: a case study using the National Surgical Quality Improvement Program (NSQIP) database. Ann Surg 2005; 242: 463-8; discussion 468-71.

6. Mahajan RP. Critical incident reporting and learning. $\mathrm{Br} \mathrm{J}$ Anaesth 2010; 105: 69-75.

7. Office NA: Managing the costs of clinical negligence in trusts. Available from URL: https://www.nao.org.uk/report/managingthe-costs-of-clinical-negligence-in-trusts/ (accessed March 2018).

8. Kim F, da Silva r, Gustafson D, Nogueira L, Harlin T, Paul DL. Current issues in patient safety in surgery: a review. Patient Saf Surg 2015; 26: DOI: https://doi.org/10.1186/s13037-015-0067-4. 
9. Merry $A F$. Safety in anaesthesia: reporting incidents and learning from them. Anaesthesia 2008; 63: 337-9.

10. Flanagan JC. The critical incident technique. Psychol Bull 1954; 51: 327-58.

11. Editorial: Airline Safety and the Value of Failure. The Globe and Mail 2018.

12. Available from URL: http://www.npsf.org/page/jeffreycooper (accessed March 2018).

13. Cooper JB, Newbower RS, Long CD, McPeek B. Preventable anesthesia mishaps: a study of human factors. Anesthesiology 1978; 49: 399-406.

14. Dobson G, Chong M, Chow L, et al. Guidelines to the practice of anesthesia - revised edition 2018. Can J Anesth 2018; 65: 76-104.

15. Safety WAfP: WHO Draft Guidelines for Adverse Event Reporting and Learning Systems: from Information to Action. Geneva 2005

16. Brunsveld-Reinders AH, Arbous MS, De Vos R, De Jonge E. Incident and error reporting systems in intensive care: a systematic review of the literature. Int $\mathrm{J}$ Qual Health Care 2016; 28: 2-13.

17. Noble DJ, Pronovost PJ. Underreporting of patient safety incidents reduces health care's ability to quantify and accurately measure harm reduction. J Patient Saf 2010; 6: 247-50.
18. Hutchinson A, Young TA, Cooper KL, et al. Trends in healthcare incident reporting and relationship to safety and quality data in acute hospitals: results from the National Reporting and Learning System. Qual Saf Health Care 2009; 18: 5-10.

19. Gibbs NM, Culwick M, Merry AF. A cross-sectional overview of the first 4,000 incidents reported to webAIRS, a de-identified web-based anaesthesia incident reporting system in Australia and New Zealand. Anaesth Intensive Care 2017; 45: 28-35.

20. Guffey PJ, Culwick M, Merry AF. Incident reporting at the local and national level. Int Anesthesiol Clin 2014; 52: 69-83.

21. Williams GD, Muffly MK, Mendoza JM, Wixson N, Leong $K$, Claure RE. Reporting of Perioperative Adverse Events by Pediatric Anesthesiologists at a Tertiary Children's Hospital: Targeted Interventions to Increase the Rate of Reporting. Anesth Analg 2017; 125: 1515-23.

22. Gibbs NM, Culwick MD, Merry AF. Patient and procedural factors associated with an increased risk of harm or death in the first 4,000 incidents reported to webAIRS. Anaesth Intensive Care 2017; 45: 159-65.

23. Paul JE, Buckley N, McLean RF, et al. Hamilton acute pain service safety study: using root cause analysis to reduce the incidence of adverse events. Anesthesiology 2014; 120: 97-109.

24. CMPA. Perspective. 2018; 9: 10. 